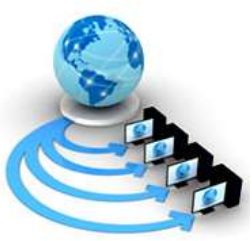

Volume 9, No. 5, September-October 2018

\title{
AN EFFICIENT ALGORITHM FOR THINNING OF IMAGES
}

\author{
Nidhi Kaushik \\ Department of Computer Science \& Engineering, \\ S.B.M.N. Engg. College, Rohtak, India
}

\author{
Vikas Raman \\ Department of Computer Science \& Applications, \\ M.D. University, Rohtak, India
}

Abstract: Thinning is an important step in image processing where we minimize (reduces) the width of the image i.e. the no. of pixels that helps in reducing the memory usage. This paper proposes a new algorithm for thinning of images and helps minimizing its size. The newly proposed algorithm preserves the connectivity of the image pattern and provides a reduced image after processing the given image. The algorithm is composed of three passes to get thinned image that is connected, reduced and gives clear view of whole image.

Keywords: Iterative, parallel, continuous, topological, geometrical.

\section{INTRODUCTION}

Thinning is a way to reduce the size of an image which helps in minimization of memory usage of the images during their processing by the machine.

- It is a mechanism of removing extra pixels in order to make it (image) just one pixel thick that reduces its size [1].

- The main purpose of thinning is to reduce the data amount required for processing an image to reduce the time taken for processing of the image and easy analysis of the patterns.

- It is an important image analysis step for its easy analysis.

- After thinning the image obtained is the binary image that we take into consideration but just a pixel thick.

- The thinning process [2], [3] can be shown below as:-

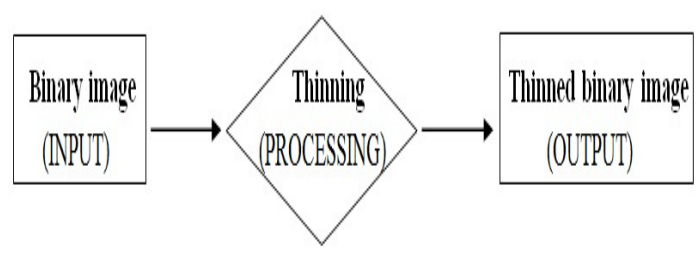

Fig 1: Thinning process

- There are various thinning algorithms given by different researchers [4]. There are broadly divided in to two categories:

(i) Iterative thinning algorithm

(a) Sequential

(b) Parallel

(ii) Non-iterative algorithm

(a) Medial axis transforms

(b) Line following

(c) Other

- The thinning process can be shown with the help of example taken in fig2.

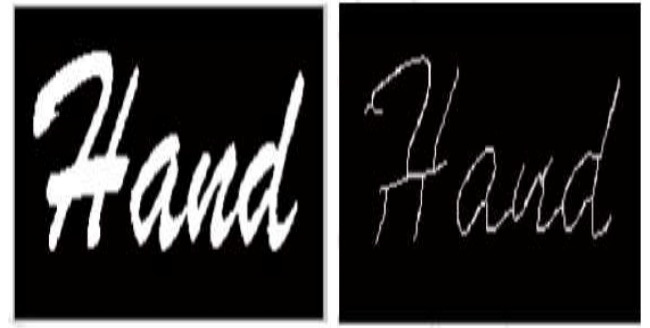

Fig 2: Thinning of an image

- This paper is organised in different sections. In the first section we give the introduction of the subject of paper thinning, its usefulness or its advantages.

- In the second section we shall discuss different thinning algorithms presented so far i.e. its literature review.

- In the third section we presented our proposed algorithm for thinning.

- In section four, we will compare the result of our proposed algorithm with other algorithms presented by other researchers.

- And in final section i.e. section five we will discuss the conclusion and future work on the thinning process.

\section{LITERATURE REVIEW}

In this section we will discuss various researches done so far on thinning process. Here we mainly discuss some usable thinning algorithms \& their strategies on thinning which are given in previous papers.

In paper [5] Waleed Abu-Ain et al. presented a thinning algorithms that merge parallel and linear approaches which is said to be iterative methods for thinning. This algorithm comprises of 3 stages where the stage one and two are basically used to extort the skeleton of the input image and the third one is used to convert the skeleton to one pixel thick image. It is likely to be a better approach on the basis of experimental results provided by the researchers. In paper [6] G.V Padole et al. proposes two thinning algorithms that are also iterative in nature for thinning of images. Here the requirement is that the image to be input for thinning should be a binary image. The first algorithm proposed by them comprises of two operations, one is to detect the edges 
and other is subtraction, where the second algorithm proposed by them is peeling off the pixels till the time the skeleton of given image is extracted. The first one is time consuming and second is an improved version. In this paper [7] Jagna and Kamakshi Prasad and presented a two pass parallel approach for thinning of binary images. This proposed algorithm makes the image just one pixel thick and preserves the connectivity of the thinned images components (pixels). The algorithm also preserves and neighbour connectivity in the binary image it shows better performance than other related algorithm and produces better quality images. In paper [8] Chatbri et al. introduced a technique to make thinning process robust against noise in the sketch of the image and it computation is also fast. It adaptively and automatically estimates the filtering scale. It is a robust technique for scratch and contour noise. In paper [9] Prakash et al. provides an algorithm on the basis of morphological operators using hyper graphs that has been applicable in biometric authentication, signature verification. The operators used here are dilation, erosion etc that acts as a filter for noise reduction.

Various iterative thinning algorithms are known till date presented by different researchers [10], [11], [12]. Here we take reference of these algorithm and design a new iterative algorithm that overcome their drawbacks. And this new algorithm is presented in the next section.

III.

PROPOSED ALGORITHM

In the proposed algorithm, we presented three passes in order to achieve thinning of an image. Here we use masking approach viz. $3 \times 3$ mask for thinning purpose. And it is applicable on every single pixel for the purpose to determine which pixels are to be selected for deletion and which reside in the resulting image.

\begin{tabular}{|l|l|l|}
\hline $\mathrm{X}_{4}$ & $\mathrm{X}_{3}$ & $\mathrm{X}_{2}$ \\
\hline $\mathrm{X}_{5}$ & $\mathrm{P}_{\mathbf{i}}$ & $\mathrm{X}_{9}$ \\
\hline $\mathrm{X}_{6}$ & $\mathrm{X}_{7}$ & $\mathrm{X}_{8}$ \\
\hline
\end{tabular}

Fig 3: Neighbours of a Pixel $P_{i}$

It is an iterative thinning algorithm that works parallel and is composed of three passes. Using it we check the continuity of the pixels and their safe deletion or retention is regulated.

The first pass in the presented algorithm is to process the pixels on the boundary at once. It checks for the conditions of deletion, if the deletion condition matches for any pixel then that pixel is deleted otherwise it resides. This will continue until we attain a two pixel wide image.

In the second pass, count the non-zero neighbours of the central pixel $\mathrm{P}_{\mathrm{i}}$. If this count is from 3 to 5 then it will further reduced to one pixel thick skeleton.

In the third pass, check for the continuity of the thinned image. If the image is continuous and satisfies the original image then stop processing otherwise make it continuous by adding pixels to the required area.

Pass-1:
Step 1: Count the non-zero neighbours and one-to-zero or zero-to-one transition of the candidate pixel $\mathrm{P}$.

Step 2: If the non-zero neighbours are from two-to-six and either on-to-zero or zero-to-nene tansitions are exactly one, then process the pixel under consideration.

Step 3: Iterate step 1 \& step 2 untili no more changes take place in pass-1, lese go to pass-2.

Pass-2:

Step 4: Count the non-zero neighbours of the candidate pixel $P_{1}$. If the count is three-to-five, then process the pixel under consideration.

Step 5: Repeat step 4 untill no moet changes take place in pass-2, else go to pass-3.

Pass-3:

Step 6: Traverse the thimned image and if there occurs any discontinuity other than the original image criteria, then add pixels to the area.

Step 7: Repeat step 6 untili no more changeses take place in pass-3.

\section{RESULTS \& COMPARISONS}

The results of the presented algorithm are compared with the existing thinning algorithms like $\mathrm{KNP}, \mathrm{ZS}$ etc. and found better in terms of connectivity, thickness, erosion, end point preservation and appearance quality. Some of the figures thinned using the proposed algorithm is shown below:

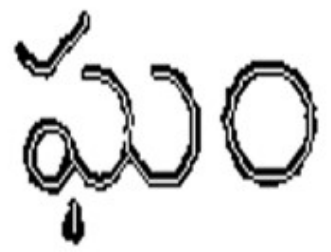

Fig 4 (a)

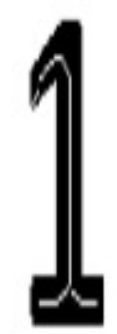

Fig 4 (b)

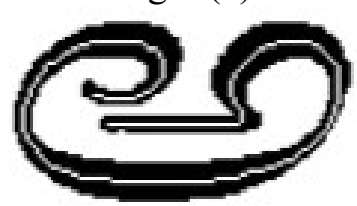

Fig 4 (c) 


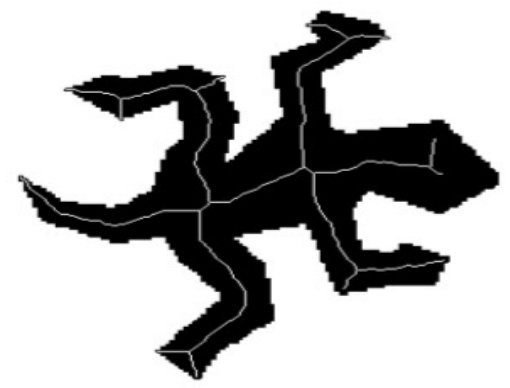

Fig 4 (d)

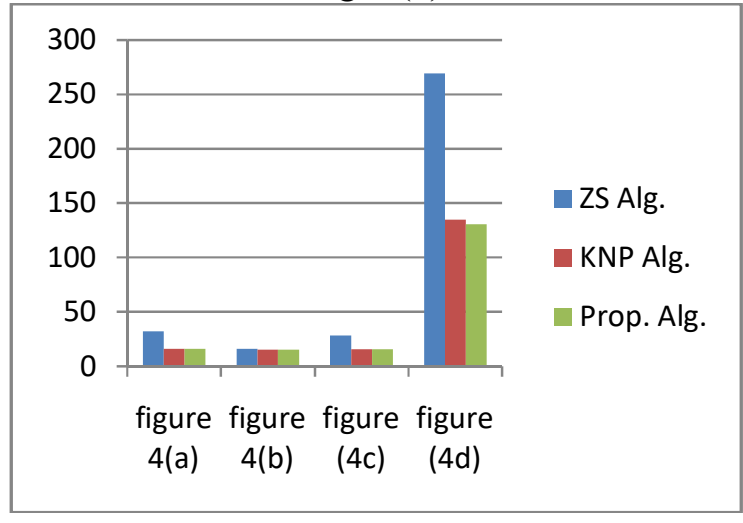

\section{CONCLUSION}

The algorithm we presented here is an efficient algorithm for thinning of images. As we compare it with other algorithms in many aspects like connectivity, image quality, end-point preservation etc and it is found to be efficient than the existing algorithms. Results after its implementation also show that it is an efficient algorithm.

\section{REFERENCES}

[1] L. Lam, S.W. Lee \& C.Y. Suen, "Thinning Methodologies - A Comprehensive Survey", IEEE Transactions on Pattern Analysis and Machine Intelligence, Vol. 14, N0. 9, Sept. 1992, pp. 869-885

[2 ]Lawrence O'Gorman \& Rangachar Kasturi, Documnet Image Analysis, IEEE Computer Society Executive Briefings, 1997

[3] A.K. Jain, Fundamentals of Digital Image Processing, Prentice Hall, 1986

[4] E. Hastings, "A Survey of Thinning Methodologies", Pattern Analysis \& Machine Intelligence, IEEE Transactions, vol. 4, Issue 9, 1992, pp. 869-885

[5] Waleed Abu-Ain, Siti Nural Sheikh Abdullah, Bilal Bataineh, Tarik Abu-Ain \& Khairuddin Omar "Skeletonization Algorithm for Binary Images", $4^{\text {th }}$ International Conference on Electrical Engineering and Informatics (2013)

[6] G.V Padole and Dr S.B Pokle ,[2010]"New Iterative Algorithms for Thin=ning Binary Images" Electronics and Tele Communication Engineering

[7] Jagna A. and Kamakshiprasad V,[April 2010] "New parallel binary image thinning algorithm" ARPN Journal of Engineering and Applied sciences

[8] H. Chatbri \& K. Kameyama, "Using Scale Space Filtering to make Thinning Algorithm Robust against Noise in Sketch Images", Pattern Recognition Letters 42(2014) pp. 1-10

[9] Prakash R.P., Keerthana S.P. and Binu V.P., "Thinning Algorithm Using Hypergraph Based Morphological Operators", Souvenir of the 2015 IEEE International Advance Computing Conference, IACC

[10] Zhang T.Y. and Suen C.Y. (1984), "A Fast Parallel Algorithm for Thinning Digital Patterns", Research Contributions, Communications of the ACM, Vol. 27, No. 3, pp. 236-239

[11] Zichang Guo \& Richard W. Hall, "Parallel Thinning with Two Sub-iteration Algorithms", Communication of ACM, vol. 32(3) March 1989, pp. 359-373

[12] A.Jagna“An efficient independent thinning Algorithm", [2014] International Journal of Advanced Research in Computer and Communication Engineering 